\title{
A New Shadow-Ring Device for Measuring Diffuse Solar Radiation at the Surface
}

\author{
Amauri P. De Oliveira and Antonio J. Machado \\ Group of Micrometeorology, Department of Atmospheric Sciences, Institute of Astronomy, Geophysics and Atmospheric Sciences, \\ University of São Paulo, Sao Paulo, Brazil \\ JoÃo F. ESCOBEDO \\ Laboratory of Solar Radiation, Department of Environmental Sciences, State University of São Paulo, Botucatu, Brazil
}

(Manuscript received 21 May 2001, in final form 4 September 2001)

\begin{abstract}
A new shadow-ring device for measuring diffuse solar radiation at the surface is presented. In this device the seasonal variation of shadow is followed by moving the detector horizontally. This unique characteristic facilitates its application for long and continuous periods of time. The blocking effect caused by the ring and other related geometric properties are formulated considering the diffuse solar radiation isotropic. The correction factor, shadow size, and ring-detector distance are derived as a function of radius and width of the ring, sun position, and local latitude. The largest blocking occurs during summer, when the ring-detector distance and the shadow width are the smallest, and it is compensated by a smaller blocking effect in the winter period. The performance of the new device is verified comparing daily values of diffuse solar radiation measured simultaneously with a similar device from Kipp \& Zonen, Inc. The results show a very good agreement (within 2.5\%) between both devices. The new device was also able to reproduce the radiometric properties of the local atmosphere based on 3-yrlong measurements of direct solar radiation using a pyrheliometer. The new device can be applied to estimate daily values of diffuse solar radiation at the surface in the range of $30^{\circ} \mathrm{N}-30^{\circ} \mathrm{S}$ with results comparable to other similar apparatuses.
\end{abstract}

\section{Introduction}

The diffuse component of the solar radiation over a horizontal surface can be estimated by blocking the direct component of solar radiation. The most common device used to block the direct component is the shadow ring. It is composed of a ring, or band, arranged to cast a shadow over the detector, which in turn collects only the diffuse component of solar radiation (Robinson and Stoch 1964; Drummond 1964; LeBaron et al. 1980; Steven and Unsworth 1980; Stanhill 1985; Sirén 1987; Michalsky et al. 1987; Battles et al. 1995).

The major advantage of the shadow-ring is that it is simple to operate and provides a direct estimate of the solar radiation diffuse component. The major disadvantage is the systematic underestimation of the diffuse solar radiation due to blocking effect caused by the ring (Steven and Unsworth 1980; LeBaron et al. 1990). This effect is difficult to take into consideration because it depends not only on the geometrical characteristic of the device, but also on the hemispherical distribution of

Corresponding author address: Dr. Amauri P. Oliveira, Group of Micrometeorology, Department of Atmospheric Sciences, Institute of Astronomy, Geophysics and Atmospheric Sciences, University of São Paulo, Rua do Matão 1226, São Paulo 05508900, Brazil.

E-mail: apdolive@usp.br solar radiation (Steven 1984; Burek et al. 1988). The combination of these two factors makes the measurements of diffuse solar radiation an observational challenge.

Correction of diffuse solar radiation measured under the shadow $\left(E_{\mathrm{DF}}^{*}\right)$ is estimated considering the ratio of blocked part $\left(E_{b}\right)$ to actual value of diffuse solar radiation $\left(E_{\mathrm{DF}}\right)$, according to the following relation:

$$
\frac{E_{\mathrm{DF}}}{E_{\mathrm{DF}}^{*}}=\left(1-\frac{E_{b}}{E_{\mathrm{DF}}}\right)^{-1} .
$$

Expression (1) is known as correction factor $\left(F_{C}\right)$ and it can be estimated analytically considering the diffuse solar radiation isotropic.

Since the field of diffuse solar radiation is seldom isotropic, the use of shadow-ring devices call for a more general and suitable way of taking into consideration the effect caused by the anisotropy on the measurements carried out with this kind of device. For instance, horizontal variation of surface albedo and uneven distribution of clouds are the more important sources of anisotropy (Battles et al. 1995). The inclusion of other effects, such as anisotropy and multiple reflections from elements of the ring, in the correction factor can only be done numerically and, most of the time, is heavily 


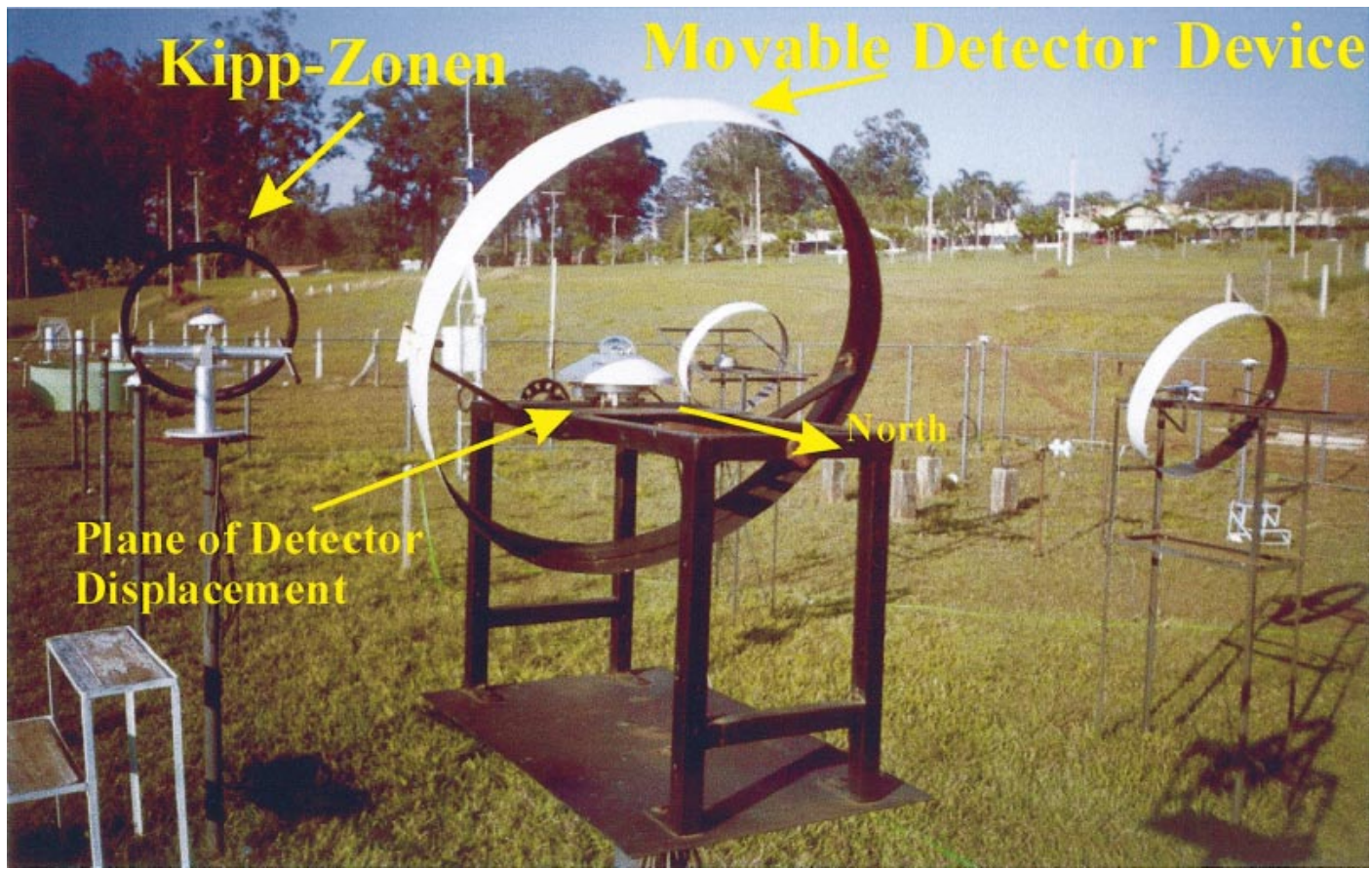

FIG. 1. View of the shadow-ring devices used in Botucatu, Brazil. The device in the foreground is an MDD; behind that is a KZD. The detector in the MDD is displaced manually in the north-south direction.

dependent on empirical expressions (LeBaron et al. 1980; Steven 1984; Rawlins and Readings 1986; LeBaron et al. 1990; Battles et al. 1995).

Despite all these difficulties, devices using shadow rings are easier to operate than others that require special setups to track the sun position, such as pyrheliometers or even shadow disks. Even though they are more sophisticated, tracking devices provide better estimates of diffuse solar radiation only under clear sky conditions. Under totally cloudy conditions the shadow-ring devices offer comparable results (Ineichen et al. 1983). Besides, the shadow-ring devices require minimal adjustment to compensate the annual evolution of shadow size and position; therefore, they can be operated continuously for a long period of time, as required for climate studies. Moreover, because of their simplicity, the shadow-ring devices cost much less than other more sophisticated devices.

Most diffuse solar radiation measurements available worldwide are based on shadow-ring devices proposed by Robinson (Robinson and Stoch 1964) or Drummond (Drummond 1964). The device proposed by Robinson is composed of a set of rings covering different celestial sphere sectors. In Drummond's device, one single ring is displaced along the earth's axis direction to compensate the annual variation of the solar declination. In both cases the detectors are kept fixed.

Recently, a new shadow-ring device, named the movable detector device (MDD), has been developed in the Laboratory of Solar Radiation at the State University of São Paulo in Botucatu, Brazil (UNESP) (Melo 1993;
Escobedo et al. 1997). In this new device the ring is fixed and the annual variation of shadow position is followed by displacing the detector horizontally (Fig. $1)$. The ring is sloped northward with an angle equal to the local latitude, and the detector is displaced manually, in a horizontal plane, by a screw mechanism that allows it to be centralized under the shadow cast by the ring. This unique characteristic makes the MDD simpler to operate than other similar devices.

Although the MDD has been in continuous operation in Brazil since 1994, in two experimental sites located in a rural area of Botucatu $\left(22^{\circ} 51^{\prime} \mathrm{S}\right)$ and in an urban area of São Paulo $\left(23^{\circ} 32^{\prime} \mathrm{S}\right)$, its properties have not been fully addressed in the literature (Escobedo et al. 1997; Oliveira et al. 2002).

Therefore, the main goal of this paper is to describe the MDD and verify its performance. A detailed derivation of the expressions used to correct the blocking effect and other MDD relevant properties are presented in section 2. The seasonal evolution for correction factor, ring-detector distance, and shadow size are presented in section 3 by comparing the behavior of the MDD with similar devices proposed by Drummond and Robinson. The effects of latitude upon these parameters are discussed in section 4, showing how the MDD behaves in a site located in the Southern Hemisphere. Two performance tests for the MDD are described in section 5. In the first one, 192 daily values of diffuse solar radiation measured with the MDD are compared with equivalent measurements using a Kipp \& Zonen, Inc., device as reference. The second test compares the MDD capacity 


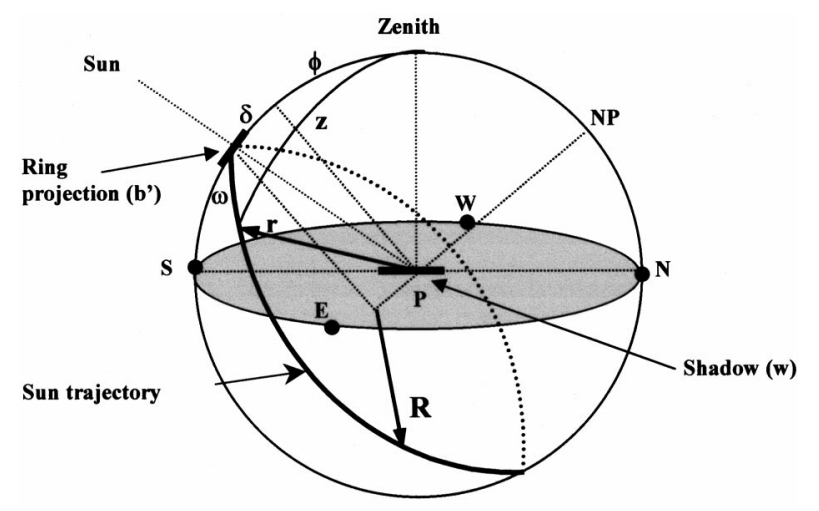

FIG. 2. Coordinates of the sun trajectory during a typical winter day for a shadow-ring device located in the Northern Hemisphere. The ring projection over the celestial sphere is indicated by $b^{\prime}$. The shadow of the ring at the solar meridian is indicated by $w$. Detector position is indicated by $P$. The other variables are solar declination $(\delta)$, local latitude $(\phi)$, solar hour angle $(\omega)$, solar zenithal angle $(z)$, radius of the celestial sphere $(r)$, and the radius of the circle formed by the sun trajectory $(R)$.

to match the radiometric properties of the atmosphere based on three years of observations of global and direct components of solar radiation at the surface.

\section{Shadow-ring device}

The common feature of all shadow-ring devices is the trajectory described by the Sun's direct beam on the celestial sphere during daytime. The trajectory corresponds to a small circle with radius $R$ over a sphere of radius $r$ centered in the point $P$ (Fig. 2). To cast a shadow in point $\mathrm{P}$ all shadow rings will have to contain the trajectory described by the sun's direct beam on the celestial sphere. Their shadows must also be wide enough to cover the detector for at least one day.

Assuming that the diffuse component of the solar radiation field is isotropic, the total diffuse irradiance incoming from the atmosphere and intercepted by the surface can be written as $E_{\mathrm{DF}}=\pi I$, where $I$ is the diffuse component of the solar radiance and $\pi$ is 3.14.

Under these conditions, the amount of energy per unit of area and time, associated to the diffuse component of solar radiation blocked by a ring of width $b$, is given by

$$
E_{b}=\int I \cos (z) d \Omega,
$$

where $z$ is the solar zenithal angle and $d \Omega$ is the element of solid angle for the ring, given by

$$
d \Omega=d S / r^{2},
$$

where $E_{\mathrm{DF}}$ is the element of area of the ring.

Considering the geometric proprieties of the celestial sphere (Table 1), the area ring element can be written as

$$
d S=b r \cos (\delta) d \omega,
$$

where $\omega$ is the solar hour angle and $\delta$ is the solar declination.

\section{a. Movable detector device}

In the MDD, the ring is fixed and the detector is displaced horizontally in the north-south direction (see the plane of displacement in Fig. 3a). In this device the ring is sloped northward at an angle equal to the local latitude. The detector is moved in the plane of displacement by a screw mechanism that allows it to be centralized under the shadow of the ring. This displacement

TABLE 1. Summary of parameters for the MDD, and the Drummond and Robinson devices. The parameters in the first column are ringdetector distance $(r)$, ring width projection on the celestial sphere $\left(b^{\prime}\right)$, ring area element $(d S)$, element of ring solid angle $(d \Omega)$, energy flux of diffuse component of solar radiation blocked by the ring $\left(E_{b}\right)$, fraction of diffuse solar radiation blocked by the ring $\left(E_{b} / E_{\mathrm{DF}}\right)$, and shadow size $(w)$.

\begin{tabular}{lllc}
\hline \hline & \multicolumn{1}{c}{ MDD (Fig. 3a) } & Drummond (Fig. 3b) & Robinson (Fig. 3c) \\
\hline$r$ & $\frac{R}{\cos (\delta)}\left[\frac{\cos (\phi) \cos (\delta)}{\cos (\phi+\delta)}\right]$ & $\frac{R}{\cos (\delta)}$ & $R$ \\
$b^{\prime}$ & $b \cos (\delta)$ & $b \cos (\delta)$ & $b$ \\
$d S$ & $b R \cos (\delta) d \omega$ & $b R \cos (\delta) d \omega$ & $b r \cos (\delta) d \omega$ \\
$d \Omega$ & $\frac{b}{R} \cos (\delta)\left[\frac{\cos (\phi+\delta)}{\cos (\phi)}\right]^{2} d \omega$ & $\frac{b}{R}[\cos (\delta)]^{3} d \omega$ & $\left(\frac{b}{r}\right) \cos (\delta) d \omega$ \\
$E_{b}$ & $2 I\left(\frac{b}{R}\right) \cos (\delta)\left[\frac{\cos (\phi+\delta)}{\cos (\phi)}\right]^{2} \int_{0}^{\omega_{p}} \cos (z) d \omega$ & $2 I\left(\frac{b}{R}\right)[\cos (\delta)]^{3} \int_{0}^{\omega_{p}} \cos (z) d \omega$ & $2 I\left(\frac{b}{R}\right) \cos (\delta) \int_{0}^{\omega_{p}} \cos (z) d \omega$ \\
$\frac{E_{b}}{E_{\mathrm{DF}}}$ & $\left(\frac{2 b}{\pi R}\right) \cos (\delta)\left[\frac{\cos (\phi+\delta)}{\cos (\phi)}\right]^{2} \int_{0}^{\omega_{p}} \cos (z) d \omega$ & $\left(\frac{2 b}{\pi R}\right)[\cos (\delta)]^{3} \int_{0}^{\omega_{p}} \cos (z) d \omega$ & $\left(\frac{2 b}{\pi R}\right) \cos (\delta) \int_{0}^{\omega_{p}} \cos (z) d \omega$ \\
$w$ & $\frac{b \cos (\delta)}{\cos (\phi+\delta)}$ & $\frac{b \cos (\delta)}{\cos (\phi+\delta)}$ & $\frac{\cos (\phi+\delta)}{b}$ \\
\hline
\end{tabular}


(a) Movable Detector Device

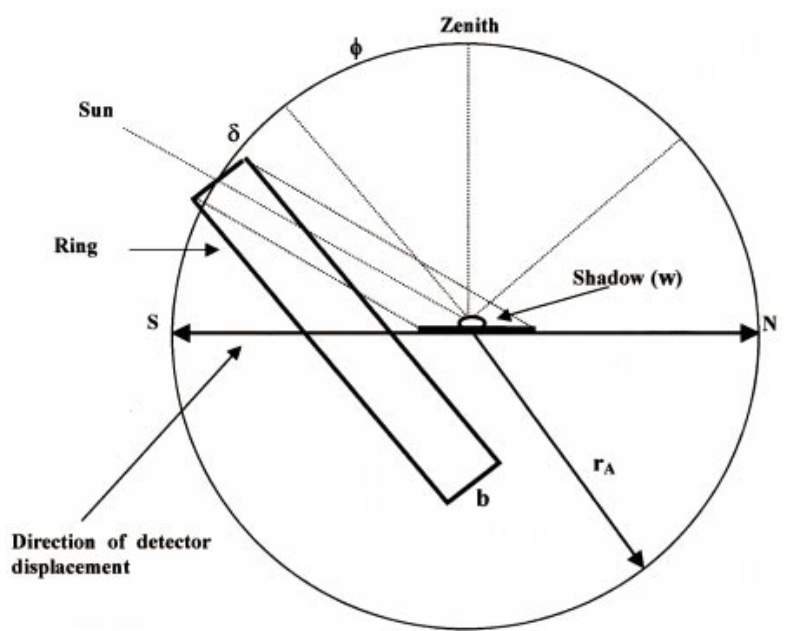

(c) Robinson's Device

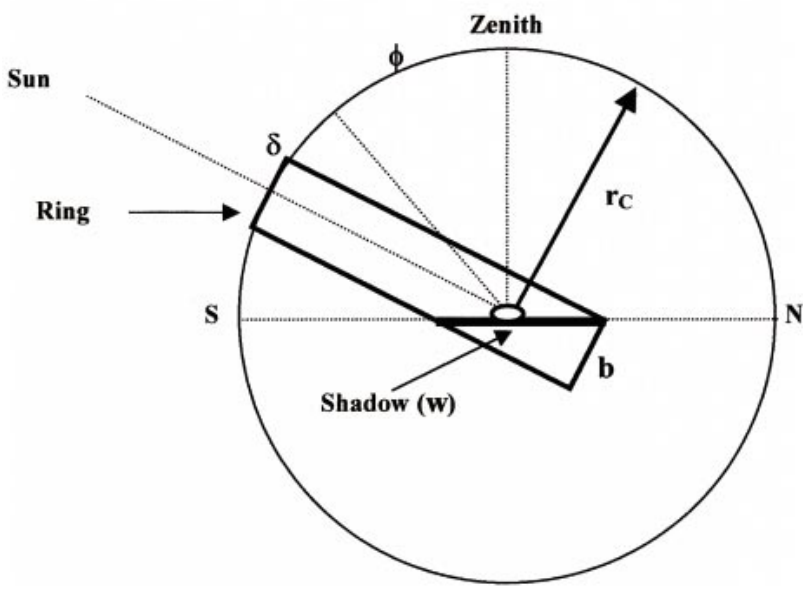

(b) Drummond's Device

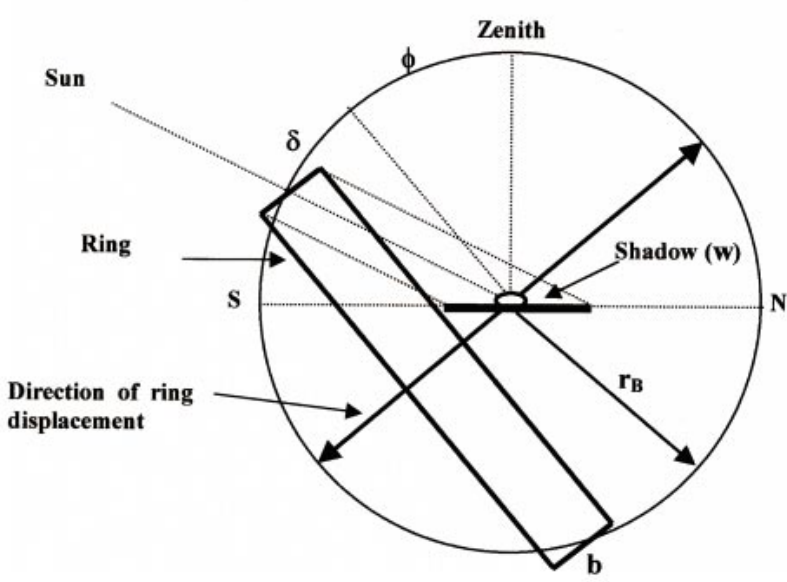

(d) MDD and Drummond's Device

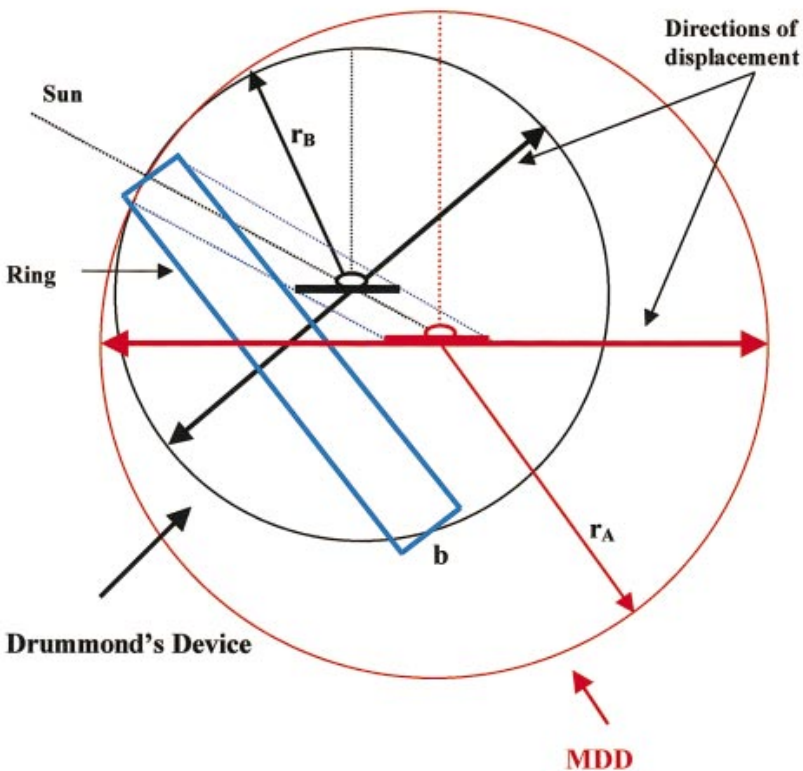

FIG. 3. Schematic representation of the shadow cast by the ring of the (a) MDD, (b) Drummond device, (c) Robinson device, and (d) MDD and Drummond device. The sun position corresponds to a winter day. The shadow rings are located in a Northern Hemisphere latitude $(\phi)$.

is carried out manually, and the position of the detector relative to the shadow is verified visually.

The only adjustments required to operate this device are the alignment of the sensor displacement in the north-south direction positioning of the ring so that it slopes northward at an angle equal to the local latitude. Given the fact that the diffuse solar radiation field is assumed isotropic, even if the detector is not perfectly centralized under the shadow, the properties derived here for the MDD will still be valid.

The effect of anisotropy certainly affects the diffuse solar radiation measurements in shadow-ring devices. Even if the detector is kept under the shadow of the MDD, there is a source of error induced by the anisotropy portion of the diffuse solar radiation component. This type of error is also present in the other devices (e.g., that of Kipp \& Zonen, Inc.). This problem will be addressed in another future work.

The radius of the sphere that intercepts the central circle of the ring [and is centered in the detector (Fig. $3 a)$ ] is equal to

$$
r_{A}=R\{1+[\sin (\delta) \sin (\phi)] / \cos (\delta+\phi)\} / \cos (\delta),
$$


where $\phi$ is the local latitude. This radius defines the distance ring detector.

The element of area in this case is given by

$$
d S=b R \cos (\delta) d \omega,
$$

while the element of solid angle is

$$
d \Omega=\frac{b}{R} \cos (\delta)\left[\frac{\cos (\phi+\delta)}{\cos (\phi)}\right]^{2} d \omega .
$$

The amount of diffuse solar radiation intercepted by the ring is given by

$$
E_{b}=2 I\left(\frac{b}{R}\right) \cos (\delta)\left[\frac{\cos (\phi+\delta)}{\cos (\phi)}\right]^{2}\left[\int_{0}^{\omega_{p}} \cos (z) d \omega\right],
$$

where $\omega_{P}$ is a half-day.

The fraction intercepted by the ring is

$$
\frac{E_{b}}{E_{\mathrm{DF}}}=\left(\frac{2 b}{\pi R}\right) \cos (\delta)\left[\frac{\cos (\phi+\delta)}{\cos (\phi)}\right]^{2}\left[\int_{0}^{\omega_{p}} \cos (z) d \omega\right] .
$$

The width of the shadow cast by the ring can be estimated by the projection of $b^{\prime}$ (Fig. 2) over the horizontal plane (Fig. 3a) and expressed as

$$
w=[b \cos (\delta)] / \cos (\phi+\delta) .
$$

Finally, the expression used to correct the blocking effect in the MDD is

$$
\begin{aligned}
F_{C}=\{1 & -\left(\frac{2 b}{\pi R}\right) \cos (\delta)\left[\frac{\cos (\phi+\delta)}{\cos (\phi)}\right]^{2} \\
& \left.\times\left[\int_{0}^{\omega_{p}} \cos (z) d \omega\right]\right\}^{-1} .
\end{aligned}
$$

\section{b. The Drummond and Robinson devices}

As mentioned before, most of diffuse solar radiation measurements available worldwide are based on shadow-ring devices proposed by Robinson or Drummond. The device proposed by Robinson is composed of a set of rings covering different celestial sphere sectors (Robinson and Stoch 1964). In Drummond's device, one single ring is displaced along the earth's axis direction to compensate the annual variation of the solar declination (Drummond 1964). In both cases the detectors are kept fixed and the rings are moved (Drummond) or replaced by others (Robinson). Therefore is worthwhile to look at the properties of these two devices and compare them with the MDD.

In the Drummond device (Fig. 3b), the ring is displaced along the earth's axis to compensate the annual variation of the sun declination. This characteristic simplifies the construction and the operation of this device. In this case, the correction factor is

$$
F_{C}=\left\{1-\left(\frac{2 b}{\pi R}\right)[\cos (\delta)]^{3} \int_{0}^{\omega_{p}} \cos (z) d \omega\right\}^{-1} .
$$

In the Robinson device the blocking procedure is accomplished by a set of rings corresponding to "slices" of a sphere with radius $r$ and centered on the detector. The ring-sensor distance does not vary as the sun changes its trajectory during the year. This characteristic simplifies the expression to correct the blocking effect (13). On the other hand, this device requires a continuous replacement of the rings to take into account the annual evolution of the solar declination. The correction factor is

$$
F_{C}=\left\{1-\left(\frac{2 b}{\pi R}\right) \cos (\delta) \int_{0}^{\omega_{p}} \cos (z) d \omega\right\}^{-1} .
$$

The expressions used in the derivation of the correction factors (12) and (13) are indicated in Table 1.

\section{c. The MDD and Drummond's device}

Visually, the relative movements of the detector with respect to the ring for the MDD (Fig. 3a) and Drummond's device (Fig. 3b) seem to be similar. In both devices the axes of the ring are oriented along the earth's axis (in the north-south direction) and the planes defined by the rings are parallel to the earth's equator in the celestial sphere (Fig. 2).

However, when these two devices are overlaid, as indicated in Fig. 3d, the important differences between them become more clear. In the MDD, the detector is displaced in the horizontal plane, as indicated by the red double arrow horizontal line in Fig. 3d. In the Drummond device, the ring is displaced in the direction of the earth's axis, as indicated by the black double arrow in Fig. 3d. The differences in the ring-detector distances $\left(r_{B}\right.$ and $\left.r_{A}\right)$ are clearer when the circles (red and black) are compared (Fig. 3d). The impact of the ring is less intense in the case of the MDD in the situation depicted in Fig. 3b, because for winter conditions the ring-detector distance is larger than in the case of the Drummond device. A more detailed comparison between these devices, including Robinson's device, will be done in section 3 .

From the practical point of view, the MDD has advantages when compared with the other two devices discussed here. It requires only an initial alignment in the north-south direction and an adjustment of the ring angle to the local latitude. The operation is easily accomplished by keeping the detector under the shadow. This can be carried out manually without any adjustment of ring angle or alignment direction.

\section{Seasonal evolution of MDD correction factor}

Annual variation of correction factor for the MDD has a larger amplitude compared to the Drummond and Robinson devices (Fig. 4a). In this comparison, all three 

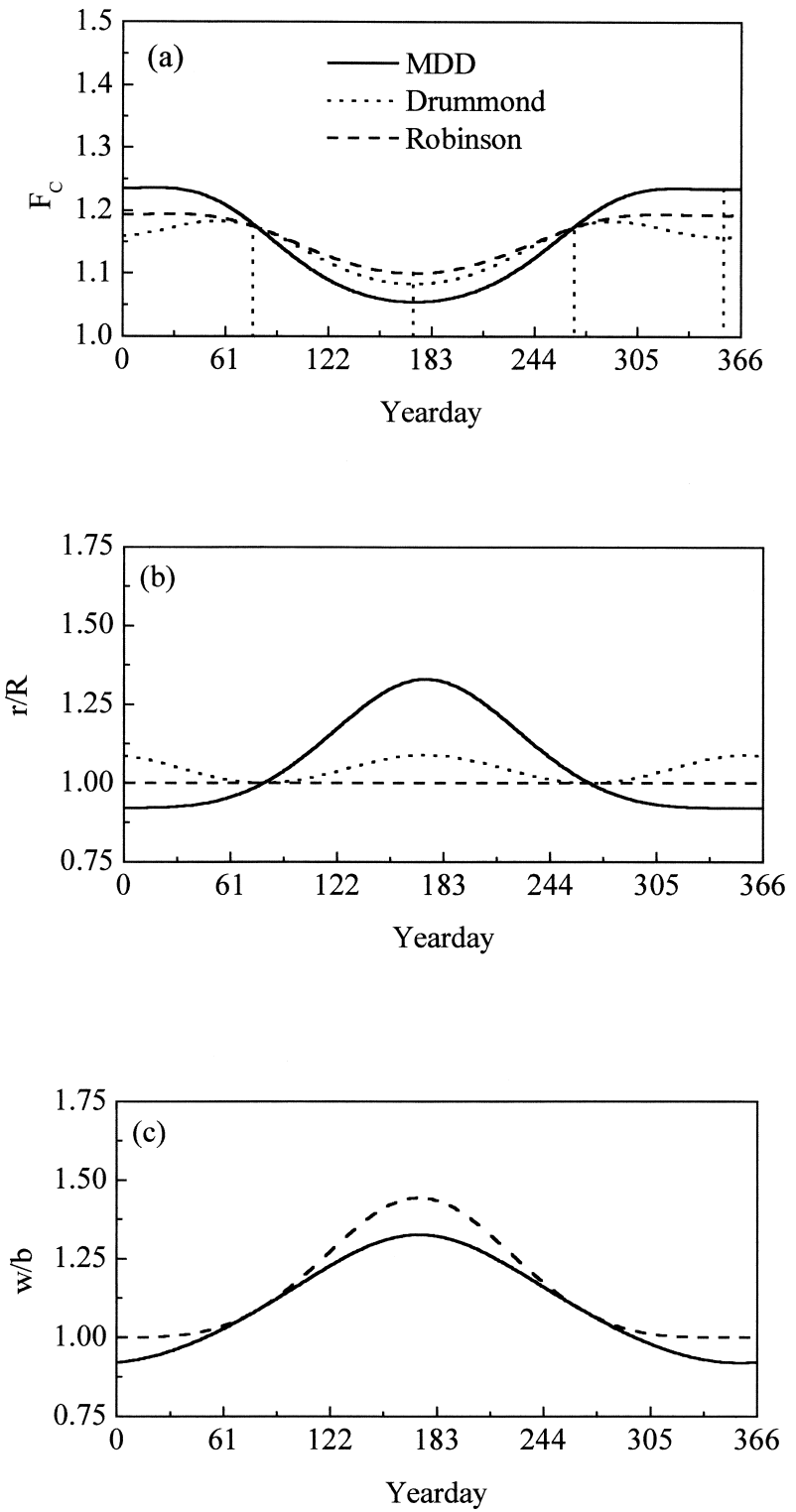

FIG. 4. Seasonal evolution of (a) correction factor, $F_{C}$; (b) ringdetector distance normalized by the ring radius, $r / R$; and (c) shadow width normalized by the ring width, $w / b$, for the MDD, and the Drummond and Robinson devices. The results are valid for the latitude of Botucatu $\left(22^{\circ} 51^{\prime} \mathrm{S}\right)$. Vertical dotted lines in (a) correspond to the equinoxes and solstices.

devices are hypothetically setup with their rings' radii and widths equal to 24 and $6 \mathrm{~cm}$, respectively. The latitude used in this comparison is $22^{\circ} 51^{\prime} \mathrm{S}$, corresponding to the local latitude where the diffuse solar radiation has been measured by the MDD. All the observational results presented in this work were gathered in this place.

The geometric properties of the three devices (Table 1) were derived based on the Northern Hemisphere geographic and astronomical characteristics (Figs. 2 and 3a-d). These expressions can be applied to estimate the properties of these three devices in the Southern Hemisphere by changing only the latitude $(\varphi)$ and solar declination $(\delta)$ signals. The results shown hereafter are applications of these expressions for the Southern Hemisphere.

The correction factor for the MDD (Fig. 4a) varies from a maximum of 1.236 in the summer (yeardays 19 and 328) to a minimum of 1.054 in the winter solstice (yearday 173). Similarly, the Robinson device shows two maximum values of 1.194 during summer (yeardays 30 and 317) and one minimum of 1.099 in the winter solstice. In the case of Drummond, the correction factor varies from a maximum value of 1.183 in the summer (yeardays 57 and 290) to a minimum value of 1.083 in the winter solstice. In the equinoxes all three devices reach the same value, 1.174 (yeardays 80 and 267).

This behavior is related to the annual evolution of ringdetector distance for the MDD and Drummond's device $\left(r_{A}\right.$ and $r_{B}$; Fig. 3d). For the MDD, the ratio $r_{A} / R$ varies from $0.92\left(r_{A}=22.1 \mathrm{~cm}\right)$ in the summer to $1.33\left(r_{A}=\right.$ $31.9 \mathrm{~cm}$ ) in the winter. For the Drummond device, $r_{B} /$ $R$ varies from $1.09\left(r_{B}=26.13 \mathrm{~cm}\right)$ in the winter and summer solstices to $1\left(r_{B}=24 \mathrm{~cm}\right)$ in the equinoxes. In the Robinson device, the annual evolution of $F_{C}$ is determined only by the relative projection of $b$ over the horizontal plane ( $r_{C}$ in Fig. 3c) since the ratio $r_{C} / R$ remains constant (equal to 1) all year long (Fig. 4b).

Another important feature of these devices is the size of the shadow cast by the ring over the horizontal plane (Fig. 4c). This parameter will determine the operational autonomy (time without manual adjustment) of the shadow ring. The annual evolution of the size of the shadow cast by the MDD is identical to that obtained by the Drummond device. The shadow ratio $(w / b)$ varies from a maximum of $1.32(w=7.96 \mathrm{~cm})$ in the winter to a minimum of $0.92(w=5.51 \mathrm{~cm})$ in the summer. In the case of Robinson, this ratio varies from $1.44(w$ $=8.66 \mathrm{~cm})$ in the winter to $1.00(w=6 \mathrm{~cm})$ during the summer. Based on this analysis it may be concluded that the MDD has the same operational autonomy of the Drummond device.

\section{Effect of latitude on seasonal evolution of the MDD correction factor}

The annual variation of the $F_{C}$ for the MDD is indicated in Fig. 5a for latitudes varying from $0^{\circ}$ to $30^{\circ} \mathrm{S}$. At the equator the MDD behaves exactly like the Drummond device. There, $F_{C}$ for both devices shows a maximum of 1.189 in fall and spring equinoxes (yeardays 80 and 267 in Table 2) to a minimum of 1.128 in the winter and summer solstices (yeardays 173 and 356). At this latitude, the ratio of ring-detector distance to radius of the ring $(r / R$ in Fig. 5b) shows two maximum values, 1.089 in the winter solstice (yearday 173) and 1.088 in the summer solstice (yearday 356), and two minimum values in the fall and spring equinoxes (yeardays 80 and 267). The annual evolution of the shadow 

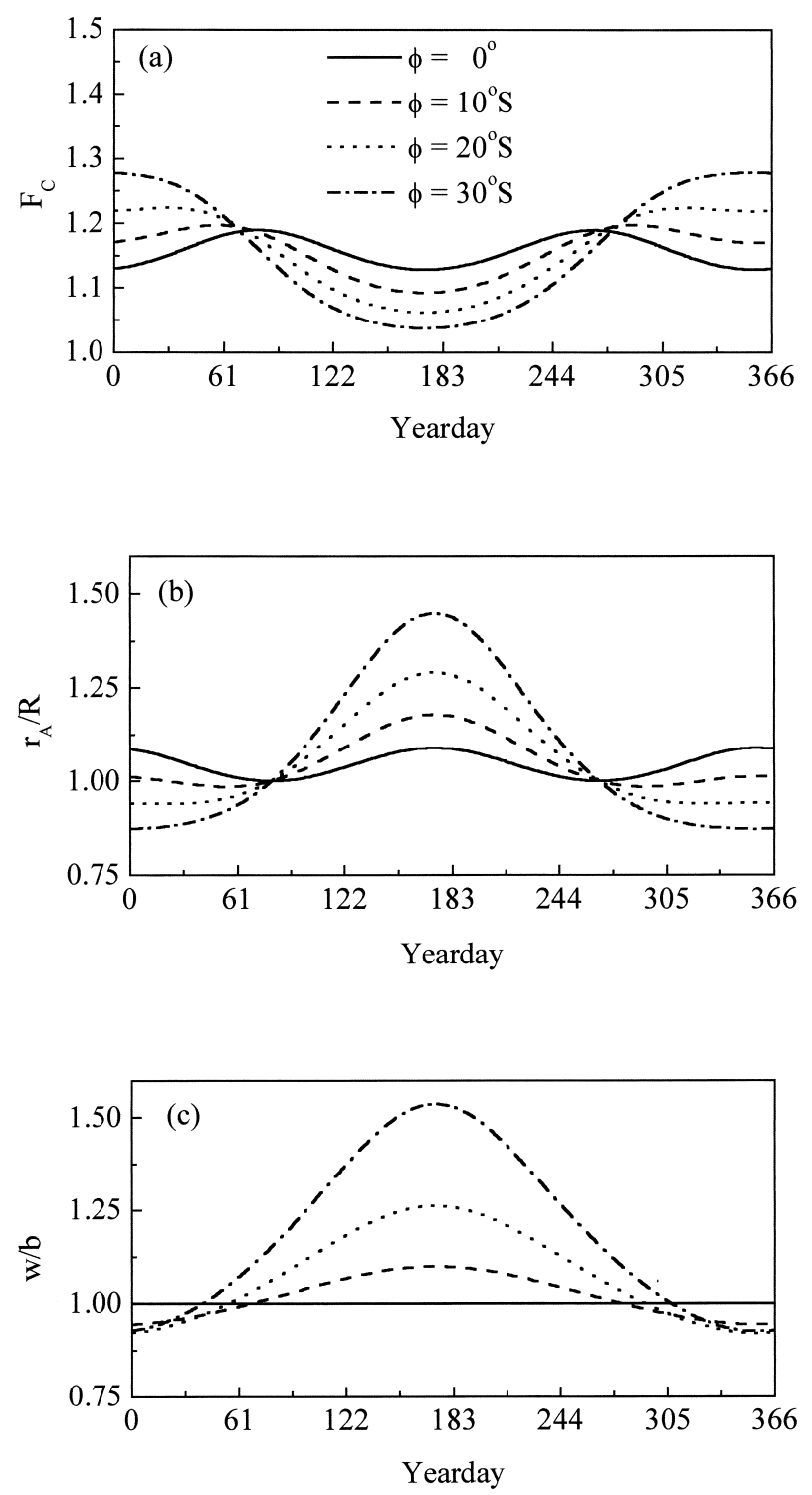

FIG. 5. Seasonal evolution of (a) correction factor, $F_{C}$; (b) ringdetector distance normalized by the ring radius, $r_{A} / R$; and (c) shadow width normalized by ring width, $w / b$, for the MDD located at $0^{\circ}, 10^{\circ}$, $20^{\circ}$, and $30^{\circ} \mathrm{S}$ latitude $(\phi)$.

width (cast by the ring on the horizontal plane) normalized by the ring width $(w / b$ in Fig. $5 c)$ indicates that the size of the shadow at the equator remains constant and equal to $b$ during the whole year.

Far from the equator the MDD behavior departs significantly from that of the other devices. The amplitude of $F_{C}$ increases and the annual distribution of maximum and minimum diverges from the pattern found at the equator (Fig. 5a). At $30^{\circ} \mathrm{S}, F_{C}$ reaches a maximum of 1.277 in the summer (yeardays 1 and 356 in Table 2) and a minimum of 1.037 in the winter solstice. At this latitude, the ratio of ring-detector distance to ring radius $\left(r_{A} / R\right.$ in Fig. 6b) varies from 0.872 in the summer to 1.448 in the winter solstice. The shadow width to ring
TABLE 2. Correction factor $\left(F_{\mathrm{C}}\right)$ (top) maximum and (bottom) minimum values as a function of yeardays. They correspond to an MDD with $b / R=0.25$ and located at latitudes $(\phi)$ varying from $0^{\circ}$ to $30^{\circ} \mathrm{S}$ (Fig. 5). The latitude of $22^{\circ} 51^{\prime} \mathrm{S}$ corresponds to Botucatu.

\begin{tabular}{lcc}
\hline \hline Latitude & $F_{C}$ & Yeardays \\
\hline Maximum & & \\
$0^{\circ}$ & 1.189 & 80 and 267 \\
$10^{\circ} \mathrm{S}$ & 1.197 & 58 and 289 \\
$20^{\circ} \mathrm{S}$ & 1.223 & 30 and 317 \\
$22^{\circ} 51^{\prime} \mathrm{S}$ & 1.236 & 19 and 328 \\
$30^{\circ} \mathrm{S}$ & 1.277 & 01 and 356 \\
$\mathrm{Minimum}$ & & \\
$0^{\circ}$ & 1.128 & 173 and 356 \\
$10^{\circ} \mathrm{S}$ & 1.092 & 173 \\
$20^{\circ} \mathrm{S}$ & 1.062 & 173 \\
$22^{\circ} 51^{\prime} \mathrm{S}$ & 1.054 & 173 \\
$30^{\circ} \mathrm{S}$ & 1.037 & 173 \\
\hline
\end{tabular}

width ratio $(w / b$ in Fig. 5c) varies from 0.925 in the summer to 1.536 in the winter solstice.

The maximum diffuse solar radiation blocking occurs during the summer period and is compensated by the smaller blocking during winter. The fact that the presence of cloud makes the diffuse solar radiation field more isotropic (Steven and Unsworth 1980) seems to indicate that the MDD is more suitable to regions where there is more cloud activity in summer than in winter. For latitudes greater than $30^{\circ}$, the behavior of $F_{C}$ for the MDD (not shown here) diverges considerably from that for other devices, and its application is not recommended.

\section{Performance of the MDD}

During 7 months the MDD and the Kipp \& Zonen device (KZD) were used to measure diffuse solar radiation at the surface in order to verify the MDD performance. These measurements took place in the city

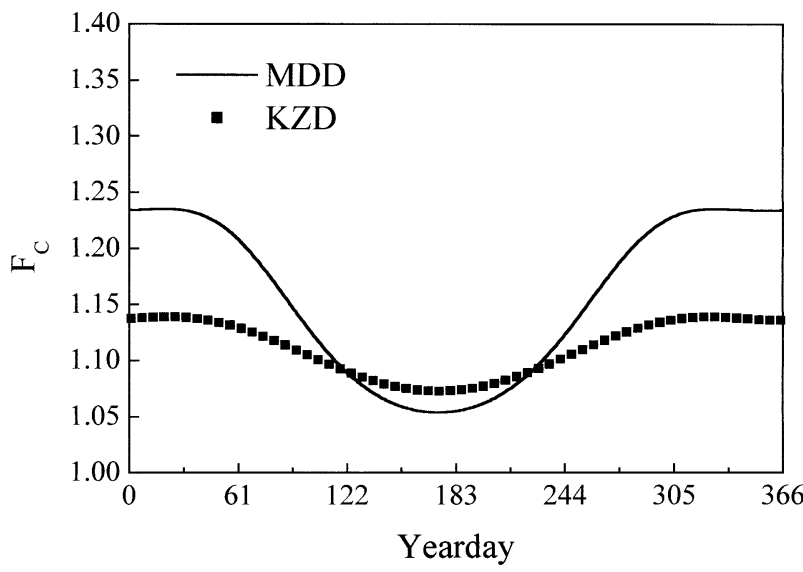

FIG. 6. Seasonal evolution of correction factor $\left(F_{C}\right)$ corresponding to the MDD and the KZD at the latitude of Botucatu $\left(22^{\circ} 51^{\prime} S\right)$. The shadow width and ring radius were set equal to 6 and $24 \mathrm{~cm}$, respectively. 


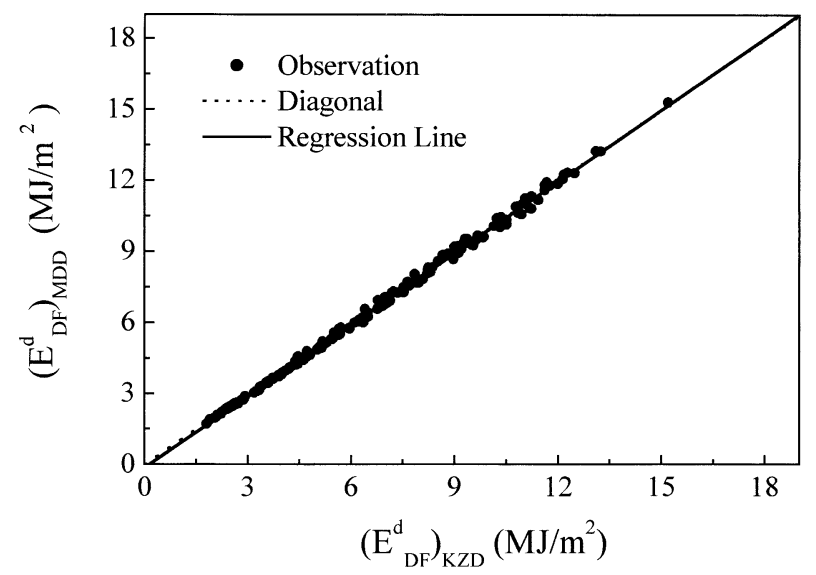

FIG. 7. Scatter diagram for diffuse solar radiation daily values measured by the MDD vs the KZD. The rings used in both devices have $b=6 \mathrm{~cm}$ and $R=24 \mathrm{~cm}$. The data corresponds to 192 days of observation in Botucatu, from 17 Dec 1999 (yearday 351) to 7 Jul 2000 (yearday 192). The continuous line corresponds to a linear regression coefficient equal to 0.99 .

of Botucatu between 17 December 1999 (yearday 351) and 11 July 2000 (yearday 192) and both devices were set up at $2 \mathrm{~m}$ above the surface. This site corresponds to a rural area characterized by homogeneous horizontally distributed green short grass surrounded by spots of deciduous forest at the south (Fig. 1).

In the MDD, the diffuse component was measured with a CM21 pyranometer model (SN 980.537) built by Kipp \& Zonen, Inc., with a calibration constant equal to $11.99 \pm 0.12 \mu \mathrm{V} \mathrm{W}^{-1} \mathrm{~m}^{-2}$. The KZD corresponds to a CM121B shadow-ring model, and the diffuse solar radiation was measured with a CM21 pyranometer model (SN 980.017) also built by Kipp \& Zonen, with a calibration constant equal to $9.93 \pm 0.10 \mu \mathrm{V} \mathrm{W}^{-1} \mathrm{~m}^{-2}$.

The geometric dimensions of the MDD were set similar to the KZD, with both rings having a radius and width equal to 24 and $6 \mathrm{~cm}$, respectively. The correction factors are indicated in Fig. 6. The smooth and continuous annual evolution of $F_{C}$ for the KZD was obtained by fitting a sixth-degree polynomial to the table of values proposed by the manufacturer. In Fig. 6 one notices that, despite the geometric similarity of both devices, their $F_{C}$ annual evolution differs significantly. The largest difference occurs during the summer period, when the ring is closest to the pyranometer in the MDD.

The comparison carried out here is based on 192 daily values of diffuse solar radiation $\left(E_{\mathrm{DF}}^{d}\right)$. All solar radiation quantities used in this part of the work are expressed in units of $10^{6}$ Joules per square meter $\left(\mathrm{MJ} \mathrm{m}^{-2}\right)$. The parameters $K_{T}$ and $K_{d}$, clearness index and diffuse fraction, respectively, were estimated as $E_{G}^{d} / E_{T}^{d}$ and $E_{\mathrm{DF}}^{d} /$ $E_{G}^{d}$. Here, $E_{G}^{d}$ corresponds to the daily value of global solar radiation at the surface (available from a model 2 pyranometer designed by Eppley, Inc.), and $E_{T}^{d}$ to the daily value of solar radiation at the top of the atmo-

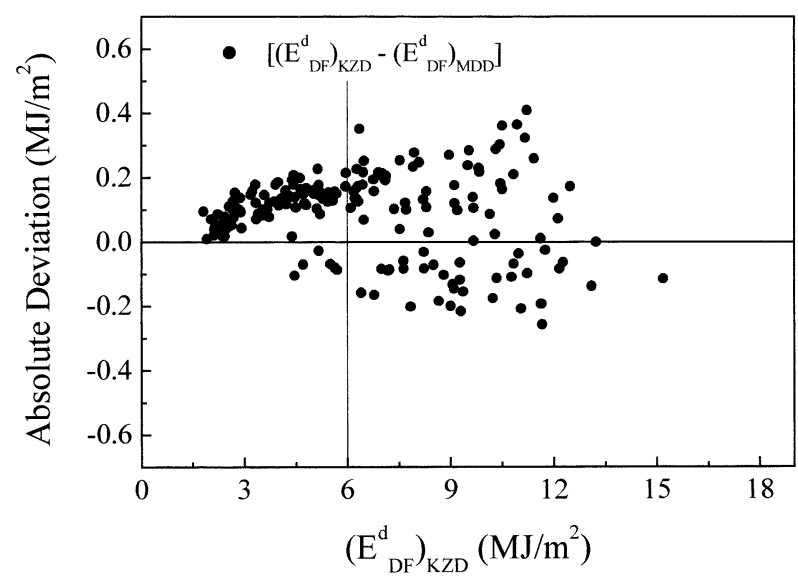

FIG. 8. Scatter diagram of absolute deviation of diffuse solar radiation daily values measured with the MDD and the $\mathrm{KZD},\left[\left(E_{\mathrm{DF}}^{d}\right)_{\mathrm{KZD}}\right.$ $\left.-\left(E_{\mathrm{DF}}^{d}\right)_{\mathrm{MDD}}\right]$, in Botucatu.

sphere, estimated theoretically considering the solar constant equal to $1366 \mathrm{~W} \mathrm{~m}^{-2}$ (Frölich and Lean 1998).

Figure 7 shows the scatter diagram of daily values of diffuse solar radiation measured by the MDD and the KZD. There one can see that despite the difference in the seasonal evolution of $F_{C}$ (Fig. 6), there is very good agreement between the measurements obtained with the two devices. The linear correlation coefficient is 0.99 , and the diagonal (dotted line) and the fitted curve obtained by linear regression (continuous line) show a small mismatch near to the diagram origin.

The scatter diagram of the absolute deviation, $A D=$ $\left[\left(E_{\mathrm{DF}}^{d}\right)_{\mathrm{KZD}}-\left(E_{\mathrm{DF}}^{d}\right)_{\mathrm{MDD}}\right]$, as a function of diffuse solar radiation daily values measured by the KZD is shown in Fig. 8. The distribution of the absolute deviation indicates that the MDD underestimates the daily values of diffuse solar radiation compared to the KZD for $E_{\mathrm{DF}}^{d}<6 \mathrm{MJ} \mathrm{m}^{-2}$, as pointed out by the vertical dotted line in Fig. 8. This behavior shows that the MDD underestimates the KZD measurements under clear sky conditions. Above this limit there is no visually detectable pattern in the distribution of absolute deviation.

Frequency distribution of relative deviation between the MDD and the KZD is shown in Fig. 9. The relative deviation, $\mathrm{RD}=100 \%\left[\mathrm{AD} /\left(E_{\mathrm{DF}}^{d}\right)_{\mathrm{KZD}}\right]$, has a distribution that is approximately Gaussian around $2.5 \%$, indicating that the MDD has a tendency to underestimate diffuse solar radiation daily values in comparison to the KZD. There is also a secondary peak around $-1.5 \%$, indicating that there is also a condition when the MDD has a tendency to overestimate $E_{\mathrm{DF}}^{d}$.

This bimodal distribution is related to the combination of climate conditions in Botucatu and the seasonal distribution of $F_{C}$. Botucatu is located in the countryside of Brazil, at $750 \mathrm{~m}$ above the mean sea level and 250 $\mathrm{km}$ away from the Atlantic Ocean. It is characterized by mild and dry winters (June-August) and warm and wet summers (December-February). The first peak 


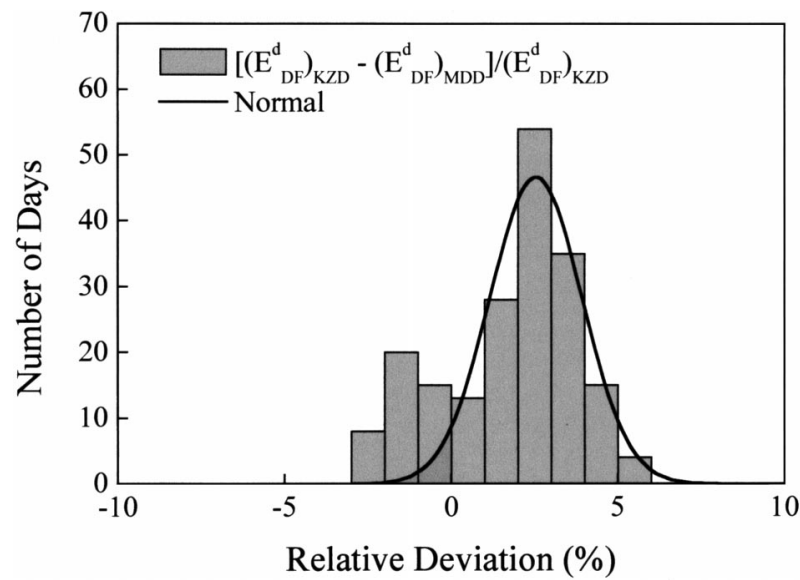

FIG. 9. Frequency distribution of relative deviation for diffuse solar radiation daily values measured with the MDD in Botucatu. The deviation was carried out considering KZD measurements as reference. The most probable relative deviation is $+2.5 \%$. Normal curve was adjusted by linear regression.

(2.5\%) is related to the larger frequency of clear sky days and partially cloudy days during the period of observation. The second peak $(-1.5 \%)$ is related to the smaller frequency of totally cloudy days.

The distribution of relative deviation as a function of the clearness index indicates that the relative deviation does not depend on the cloud cover conditions in the interval $0.25<K_{T}<0.6$ (shown in Fig. 10 by two vertical dotted lines) because the linear correlation coefficient (0.54) is not conclusive. In this interval, both positive and negative relative deviations are equally distributed. Under totally cloudy conditions $\left(K_{T}<0.30\right)$ the MDD overestimates $E_{\mathrm{DF}}^{d}$ because this condition occurs during summer period, when the solar declination is negative (Fig. 11). The correction factor for the MDD during summertime in the Southern Hemisphere is larger than that for the KZD (Fig. 6).

It is important to emphasize that the clearness index is directly proportional to the intensity of global solar radiation at the surface; therefore values of $K_{T}$ close to 1 indicate not only clear sky conditions but also larger intensity of solar radiation and vice versa. Thus, the behavior of the relative deviation with respect to $K_{T}$ can be extended to the intensity of global solar radiation at the surface.

The distribution of relative deviation as a function of solar declination is indicated in Fig. 11. The maximum relative deviation $(\sim+5 \%)$ occurs during spring and part of winter, when there is less cloud activity in Botucatu. On the other hand, the minimum relative deviation $(\sim-2.5 \%)$ occurs during summer. This behavior indicates that seasonal distribution of relative deviation results from the combined effect of seasonal variation of cloud activity and correction factor. During summer $(\delta<-10)$ there is more cloud activity, and, as explained before, the effect of the correction factor dominates the behavior of relative deviation. During spring and winter

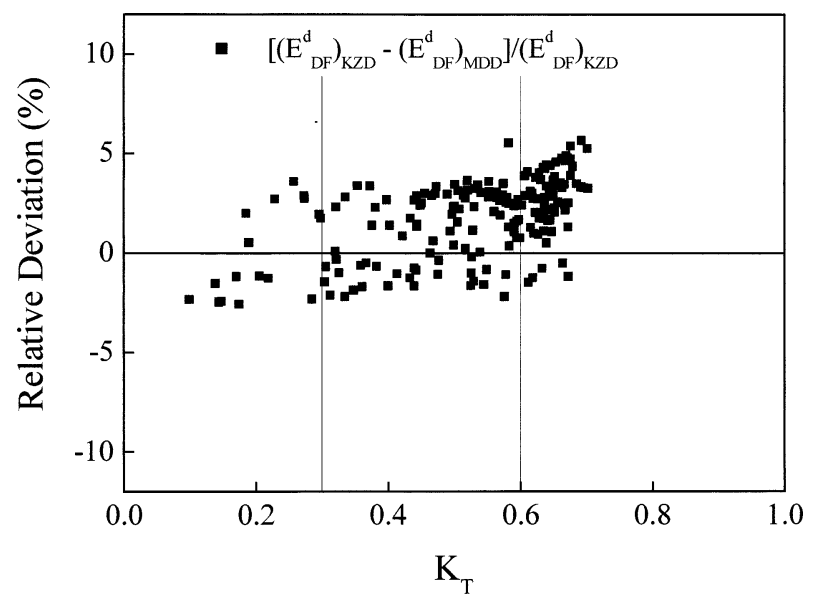

FIG. 10. Scatter diagram of relative deviation for diffuse solar radiation daily values vs clearness index $\left(K_{T}\right)$ in Botucatu.

cloud activity is smaller, and correction factors for the two devices are comparable. Therefore, the relative deviation reaches a maximum $(\sim+5 \%)$ and stays at this level. The pattern displayed in Fig. 11 is statistically significant once the linear correlation for the interpolated curve (second-degree polynomial) is equal to 0.86 .

A second performance test was undergone comparing the $K_{T}-K_{d}$ distribution obtained by the MDD with the distribution representative of the local climate. This later distribution is indicated by a curve (Fig. 12), obtained from a polynomial fitting of $K_{T}-K_{d}$ values based on 3 -yr-long measurements of direct solar radiation (using a pyrheliometer) and global solar radiation (using a pyranometer), as described by Ricieri (1999).

The values of $K_{d}$ obtained by the MDD are symmetrically distributed around the polynomial, indicating that, for $0.25<K_{T}<75$, the device is able to reproduce the climatological behavior. For $K_{T}<0.25$, the MDD

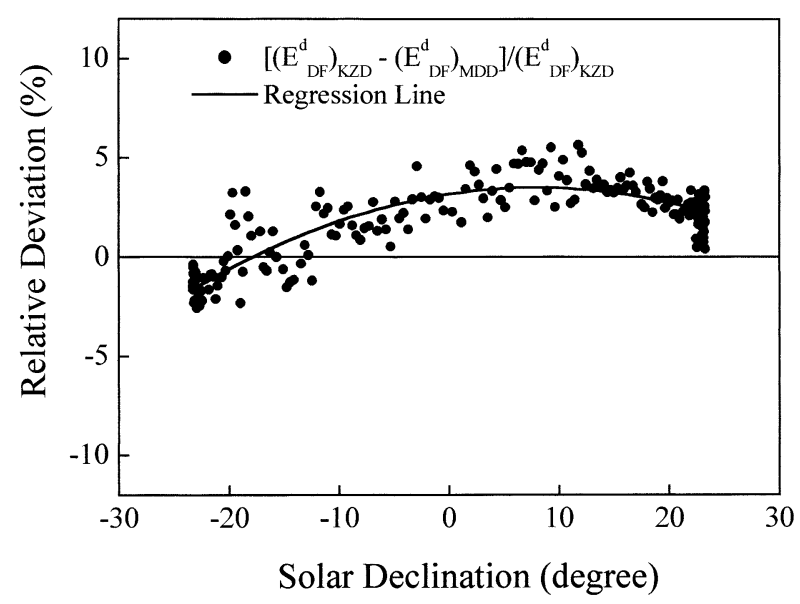

FIG. 11. Scatter diagram of relative deviation distribution for daily values of diffuse solar radiation as a function of solar declination $(\delta)$ in Botucatu. The fitted curve corresponds to a linear regression coefficient of 0.86 . 


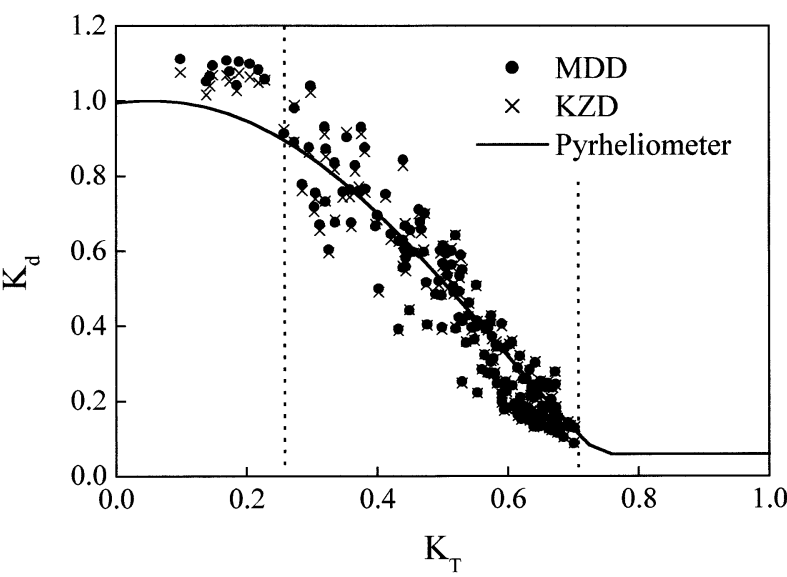

FIG. 12. A $K_{T}-K_{d}$ diagram based on diffuse solar radiation daily values measured with the MDD and the KZD in Botucatu. The solid line corresponds to the adjusted curve through the $K_{T}-K_{d}$ values obtained from diffuse solar radiation measured with a pyrheliometer during $3 \mathrm{yr}$ in Botucatu (Ricieri 1999).

yields slightly larger values of $K_{d}$ due to the correction factor effects discussed before. As expected, the $K_{T}-$ $K_{d}$ distribution obtained by the MDD is comparable with the KZD distribution.

\section{Conclusions}

In this work major features of a new shadow-ring device have been presented. This device, called the mobile detector device, was developed in the Laboratory of Solar Radiation at UNESP (Melo 1993) and has been used continuously to measure diffuse solar radiation in Botucatu (Escobedo et al. 1997) and São Paulo city since 1994 (Oliveira et al. 1996, 2002).

In the MDD the ring is fixed, and the annual variation of shadow position is followed by displacing the detector horizontally. The ring is sloped northward at an angle equal to the local latitude, and the detector is displaced manually, in a horizontal plane, by a screw mechanism that allows it to be centralized under the shadow cast by the ring. This unique characteristic makes this device simpler to operate compared to other devices in which the detector is fixed.

Considering the diffuse solar radiation isotropic, the correction factor for the MDD is given by

$$
\begin{aligned}
F_{C}=\{1 & -\left(\frac{2 b}{\pi R}\right) \cos (\delta)\left[\frac{\cos (\phi+\delta)}{\cos (\phi)}\right]^{2} \\
& \left.\times\left[\int_{0}^{\omega_{p}} \cos (z) d \omega\right]\right\}^{-1} .
\end{aligned}
$$

The ring-detector distance and shadow size are given by

$$
\begin{aligned}
r_{A} & =R\{1+[\sin (\delta) \sin (\phi)] / \cos (\delta+\phi)\} / \cos (\delta), \\
w & =[b \cos (\delta)] / \cos (\phi+\delta) .
\end{aligned}
$$

Major geometric properties of the MDD were compared with the ones for the Robinson and Drummond devices, indicating that $F_{C}$ for the MDD is higher during summer and smaller during winter. In latitudes between $0^{\circ}$ and $30^{\circ} \mathrm{S}$, the maximum value of $F_{C}$ varies from 1.189 to 1.277 , and the minimum from 1.128 to 1.037 . The highest correction factors occur during summer when the ring-detector distance and shadow width are smaller.

The comparison between diffuse solar radiation daily values measured by the new device and the Kipp \& Zonen device indicates a good agreement, with a linear correlation coefficient of 0.99 . The deviation between the MDD and the KZD is due to the combined effect of annual distribution of cloud and $F_{C}$; the relative deviation distribution varies from $-3 \%$ to $+6 \%$.

The comparison between values of $K_{d}$, obtained with both devices (MDD and KZD), shows that the performance of the prototype is similar to that of the Kipp \& Zonen device for atmospheric conditions varying from partially cloudy to totally clear $\left(0.25<K_{T}<75\right)$, while for totally cloudy sky the performance of the MDD is slightly inferior to that of the KZD.

The MDD was able to reproduce the climatological behavior obtained from a polynomial fitting of $K_{T}-K_{d}$ values based on 3-yr-long measurements of direct and global solar radiation. The results presented here indicate that the MDD can be applied, with results comparable to other similar apparatus, to estimate diffuse solar radiation daily values at the surface in the range of $30^{\circ} \mathrm{N}-30^{\circ} \mathrm{S}$.

Acknowledgments. The authors acknowledge financial support provided by "Conselho Nacional de Desenvolvimento Científico e Tecnológico-CNPq" and by "Fundação de Amparo à Pesquisa do Estado de São Paulo-Fapesp."

\section{REFERENCES}

Battles, F. J., F. J. Olmo, and L. Alados-Arboledas, 1995: On shadowband correction methods for diffuse irradiance measurements. Sol. Energy, 54, 105-114.

Burek, S. A. M., B. Norton, and S. D. Probert, 1988: Analytical and experimental methods for shadow-band correction factors for solarimeters on inclined planes under isotropically diffuse and overcast skies. Sol. Energy, 40, 151-160.

Drummond, A. J., 1964: Comments on "Sky radiation measurement and corrections." J. Appl. Meteor., 3, 810-811.

Escobedo, J. F., V. A. Frisina, M. A. Chaves, and A. P. Oliveira, 1997: Radiômetros solares com termopilhas de filmes Finos II Desempenho (in Portuguese). Rev. Bras. Aplicações Vácuo, 16, 16-21.

Frölich, C., and L. Lean, 1998: The sun's total irradiance: Cycles and trends in the past two decades and associated climate change uncertainties. Geophys. Res. Lett., 25, 4377-4380.

Ineichen, P., J. M. Grenaud, O. Guisan, and A. Mermoud, 1983: Study of the corrective factor involved when measuring the diffuse solar radiation by the use of the ring method. Sol. Energy, 31, 113-117.

LeBaron, B. A., W. A. Peterson, and I. Dirmhirn, 1980: Corrections for diffuse irradiance measured with shadowbands. Sol. Energy, 25, 1-13. 
, J. J. Michalsky, and R. Perez, 1990: A simple procedure for correcting shadowband data for all sky conditions. Sol. Energy, 44, 249-256.

Melo, J. M. D., 1993: Desenvolvimento de um sistema para medir simultaneamente radiações global, difusa e directa (in Portuguese). Tese de Doutorado, Faculdade de Ciências Agronômicas, UNESP-Botucatu, $130 \mathrm{pp}$.

Michalsky, J. J., L. Harrison, and B. A. Le Baron, 1987: Empirical radiometric correction of a silicon photodiode rotating shadowband pyranometer. Sol. Energy, 39, 87-96.

Oliveira, A. P., J. F. Escobedo, A. Plana-Fattori, J. Soares, and P. M. Santos, 1996: Medidas de radiação solar na Cidade de São Paulo: Calibração de piranômetros e aplicações meteorológicas (in Portuguese). Rev. Bras. Geofís., 14, 203-216.

— A. J. Machado, J. F. Escobedo, and J. Soares, 2002: Diurnal evolution of the solar radiation in the City of São Paulo, Brazil: Seasonal variation and modeling. Theor. Appl. Climatol., 71, 231-249.

Rawlins, F., and C. J. Readings, 1986: The shade ring correction for measurements of diffuse irradiance under clear skies. Sol. Energy, 37, 407-416.

Ricieri, R. P., 1999: Modelos de estimativa e avaliação dos métodos de medída da radiações solar difusa (in Portuguese). Tese de Doutorado, Faculdade de Ciências Agronômicas, UNESP-Botucatu, 89 pp.

Robinson, N., and L. Stoch, 1964: Sky radiation measurement and corrections. J. Appl. Meteor., 3, 179-181.

Sirén, K. E., 1987: The shadow band correction for diffuse irradiation band on a two-component sky radiance model. Sol. Energy, 39, 433-438.

Stanhill, G., 1985: Observations of shade-ring correction factors for diffuse sky radiation measurements at the Dead Sea. Quart. J. Roy. Meteor. Soc., 111, 1125-1130.

Steven, M. D., 1984: The anisotropy of diffuse solar radiation determined from shade-ring measurements. Quart. J. Roy. Meteor. Soc., 110, 261-270.

, and M. H. Unsworth, 1980: Shade-ring corrections for pyranometer measurements of diffuse solar radiation from cloudless skies. Quart. J. Roy. Meteor. Soc., 106, 865-872. 